\title{
A NEW ALGORITHM FOR THE RECURSION OF HYPERGEOMETRIC MULTISUMS WITH IMPROVED UNIVERSAL DENOMINATOR
}

\author{
STAVROS GAROUFALIDIS AND XINYU SUN
}

\begin{abstract}
The purpose of the paper is to introduce two new algorithms. The first one computes a linear recursion for proper hypergeometric multisums, by treating one summation variable at a time, and provides rational certificates along the way. A key part in the search of a linear recursion is an improved universal denominator algorithm that constructs all rational solutions $x(n)$ of the equation

$$
\frac{a_{m}(n)}{b_{m}(n)} x(n+m)+\cdots+\frac{a_{0}(n)}{b_{0}(n)} x(n)=c(n),
$$

where $a_{i}(n), b_{i}(n), c(n)$ are polynomials. Our algorithm improves Abramov's universal denominator.
\end{abstract}

\section{Contents}

1. Introduction 1

1.1. History 1

1.2. What is multivariable creative telescoping? $\quad 2$

1.3. Abramov's universal denominator algorithm 3

1.4. Acknowledgement 3

2. Two algorithms 3

2.1. A new algorithm for the recursion of hypergeometric multisums 3

2.2. An improved universal denominator algorithm 4

2.3. Plan of the proof 4

3. Use of the program and examples 5

4. Proof of the multisum algorithm 5

4.1. Two Lemmas 5

4.2. Two propositions for the algorithm $\quad 7$

4.3. Proof of Proposition $4.3 \quad 7$

4.4. Proof of Proposition 4.4 9

5. Proof of the universal denominator algorithm 9

$\begin{array}{ll}\text { References } & 11\end{array}$

\section{INTRODUCTION}

1.1. History. The paper introduces a new algorithm to find linear recursions (with coefficients polynomials in $n$ ) for multidimensional sums of the form

Date: July 8, 2009.

S.G. was supported in part by National Science Foundation.

Mathematics Classification. Primary 33F10. Secondary 05E99.

Keywords and phrases: WZ-algorithm, Creative Telescoping, Gosper's algorithm, Zeilberger's algorithm, hypergeometric, multisum, recursion, Abramov's algorithm, universal denominator. 


$$
S(n)=\sum_{k \in D} f(n, k)
$$

where $D \subset \mathbb{Z}^{r}$ and the summand $f(n, k)$ is a proper hypergeometric term in the variables $(n, k)$. By proper hypergeometric term (abbreviated by term) $f(m)$ in the variables $m=\left(m_{1}, \ldots, m_{s}\right)$ we mean an expression of the form

$$
f(m)=P(m) \prod_{j=1}^{J} A_{j}(m) !^{\epsilon_{j}}
$$

where $P(m)$ is a polynomial in $m$ and $A_{j}(m)=\sum_{i=1}^{s} a_{j i} m_{j}$ is a linear form in $m$ with integer coefficients $a_{j i}$ and $\epsilon_{j}= \pm 1$ for $1 \leq j \leq J$. Throughout this paper, $f(n, k)$ will denote a proper hypergeometric term.

As observed by Zeilberger [Ze], and further explained in [WZ], Sister Celine's method [Fas] can be used to prove the existence of linear recursions of $S(n)$ in a constructive way. A faster algorithm was constructed by Zeilberger (also known as creative telescoping [PWZ]), which employed Gosper's indefinite summation algorithm [Gos]. Creative telescoping is faster than Sister Celine's method, and often returns the optimal (i.e., minimal order) recursions. However, due to the nature of Gosper's algorithm, Zeilberger's method only works for single sums, i.e., when $r=1$ in (1).

Wegschaider in [Weg] improved Sister Celine's algorithm for multisums; Zeilberger has a program EKHAD for creative telescoping, while Paule and Schorn [PSh] implemented it in Mathematica; Schneider created a package called Sigma, the framework of which was explained in [Sch]; Apagodu and Zeilberger [AZ] generalized creative telescoping to multi-variable context which resulted in another fast algorithm.

1.2. What is multivariable creative telescoping? Multivariable creative telescoping for $S(n)$ is the problem of finding a natural number $J \in \mathbb{N}$, and rational functions $a_{j}(n) \in \mathbb{Q}(n)$, for $1 \leq j \leq J$ and rational functions $C_{i}(n, k) \in \mathbb{Q}(n, k)$ for $1 \leq i \leq r$ so that

$$
\sum_{j=0}^{J} a_{j}(n) N^{j} f(n, k)=\sum_{i=1}^{r}\left(K_{i}-1\right)\left(C_{i}(n, k) f(n, k)\right),
$$

where $N, K_{i}, n, k_{i}$ are operators that act on functions $f(n, k)$ as follows:

$$
\begin{aligned}
(N f)(n, k) & =f(n+1, k), \quad(n f)(n, k)=n f(n, k), \\
\left(K_{i} f\right)\left(n, k_{1}, \ldots, k_{r}\right) & =f\left(n, k_{1}, \ldots, k_{i-1}, k_{i}+1, k_{i+1}, \ldots, k_{r}\right), \\
\left(k_{i} f\right)\left(n, k_{1}, \ldots, k_{r}\right) & =k_{i} f\left(n, k_{1}, \ldots, k_{r}\right) .
\end{aligned}
$$

Note that the operators $N, n, K_{i}, k_{i}$ commute except in the following instance

$$
N n=n+1, \quad K_{i} k_{i}=k_{i}+1 .
$$

In Equation (3), the rational functions $C_{i}(n, k)$ for $1 \leq i \leq r$ are called the certificates and the operator $\sum_{j=0}^{J} a_{j}(n) N^{j}$ is called the recursion for the sum $S(n)$. Given Equation (3), we can sum over $k$ to obtain an inhomogeneous linear recursion for $S(n)$, whose inhomogeneous part consists of the contribution from the boundary terms.

All known algorithms of creative telescoping convert (3) to a system of linear equations with coefficients in the field $\mathbb{Q}(n, k)$. This is possible since dividing both sides of $(3)$ by $f(n, k)$ and using the fact that $f(n, k)$ is proper hypergeometric, it follows that the ratios $N f(n, k) / f(n, k) \in \mathbb{Q}(n, k)$ and $K_{i} f(n, k) / f(n, k) \in \mathbb{Q}(n, k)$ are rational functions. The number of unknowns and equations directly affect the performance of the above mentioned algorithms. 
1.3. Abramov's universal denominator algorithm. A key part of our search for a linear recursion of hypergeometric multisums is an improved universal denominator algorithm that finds all rational solutions $x(n) \in \mathbb{Q}(n)$ to a linear difference equation

$$
\frac{a_{m}(n)}{b_{m}(n)} x(n+m)+\cdots+\frac{a_{0}(n)}{b_{0}(n)} x(n)=c(n),
$$

where $a_{i}(n), b_{i}(n), c(n)$ are polynomials. The idea is to correctly predict the denominator $u(x)$ of $x(n)$ (also known as the universal denominator), so that the problem can be reduced into finding a polynomial solution to a linear difference equation. In [Ab] Abramov developed a universal denominator algorithm. In this paper, we develop a new algorithm that improves Abramov's algorithm by possibly reducing the number of factors in the universal denominator. The new algorithm is used repeatedly to convert the problem of finding recursions of multivariate hypergeometric sums into the problem of solving system of linear equations. And fewer factors in the universal denominator implies fewer numbers of variables and fewer equations in the system.

1.4. Acknowledgement. The authors wish to thank D. Zeilberger for a careful reading of an earlier version of the paper and for detailed suggestions and comments.

\section{Two ALGORIthms}

2.1. A new algorithm for the recursion of hypergeometric multisums. To describe our algorithm for the recursion of multisums, let us introduce some useful notation.

Definition 2.1. Fix a term $f(n, k)$ where $k=\left(k_{1}, \ldots, k_{r}\right)$ and $1 \leq i, j \leq r$. We say that two operators $P$ and $Q$ in the variables $n, k_{i}, N$ and $K_{i}$ are $f$-equivalent modulo $K_{i}-1, \ldots, K_{j}-1$, and write

$$
P \equiv_{f} Q \bmod \left(K_{i}-1, K_{i+1}-1, \ldots, K_{j}-1\right)
$$

if there exist rational functions $b_{s}(n, k)$ for $i \leq s \leq j$ so that

$$
(P-Q) f(n, k)=\sum_{s=i}^{j}\left(K_{s}-1\right)\left(b_{s}(n, k) f(n, k)\right) .
$$

If $i>j$, the right-hand side of the last equation is 0 .

Our algorithm will construct operators $\mathbf{R E C}_{\mathbf{i}}$ for $0 \leq i \leq r$ and $\mathbf{R E C}_{\mathbf{j}, \mathbf{i}}$ for $0 \leq i<j \leq r$ of the following form

$$
\begin{aligned}
& \mathbf{R E C}_{\mathbf{i}}=\sum_{s=0}^{d_{i}} a_{i, s}\left(n, k_{1}, \ldots, k_{i}\right) K_{i}^{s}, \quad i \neq 0 ; \quad \mathbf{R E C}_{\mathbf{0}}=\sum_{s=0}^{d_{0}} a_{0, s}(n) N^{s} \\
& \mathbf{R E C}_{\mathbf{j}, \mathbf{i}}=K_{i}+\sum_{s=0}^{d_{j, i}} a_{j, i, s}\left(n, k_{1}, \ldots, k_{j}\right) K_{j}^{s}, \quad i \neq 0 ; \quad \mathbf{R E C}_{\mathbf{j}, \mathbf{0}}=N+\sum_{s=0}^{d_{j, 0}} a_{j, 0, s}\left(n, k_{1}, \ldots, k_{j}\right) K_{j}^{s},
\end{aligned}
$$

that satisfy

$$
\mathbf{R E C}_{\mathbf{i}} \equiv_{f} 0 \bmod \left(K_{i+1}-1, \ldots, K_{r}-1\right) \quad \mathbf{R E C}_{\mathbf{j}, \mathbf{i}} \equiv_{f} 0 \bmod \left(K_{j+1}-1, \ldots, K_{r}-1\right) .
$$

We will call such operators $\mathbf{R E C} \mathbf{i}, \mathbf{R E C}_{\mathbf{j}, \mathbf{i}} f$-compatible.

Observe that $\mathbf{R E C}_{\mathbf{0}}$ is exactly Equation (3). Here are the steps for the algorithm. 
$\begin{array}{ll}\text { INPUT: } & \text { A proper hypergeometric term } f\left(n, k_{1}, \ldots, k_{r}\right) . \\ \text { OUTPUT: } & \text { A recursion } \sum_{i=0}^{I} a_{i}(n) N^{i} \text { and a set of certificates } C_{i}\left(n, k_{1}, \ldots, k_{r}\right) \text { that satisfy }(3) .\end{array}$

Step 1. Set $l:=r, k_{0}=n$ and $K_{0}:=N$;

Step 2. Set $\mathbf{R E C}_{\mathbf{r}, \mathbf{0}}:=N-\frac{N f}{f}, \mathbf{R E C}_{\mathbf{r}, \mathbf{i}}:=K_{i}-\frac{K_{i} f}{f}, 1 \leq i \leq r-1$ and $\mathbf{R E C}_{\mathbf{r}}:=K_{r}-\frac{\frac{f}{K_{r} f}}{f}$;

Step 3. Construct $\mathbf{R E C}_{\mathbf{r}-\mathbf{1}}$ using Proposition 4.4.

Step 4. $\quad$ If $l=1$, print $\mathbf{R E C}_{\mathbf{0}}$ and stop; otherwise, continue;

Step 5. $\quad$ Construct $\mathbf{R E C}_{\mathbf{l - 1 , \mathbf { i }}}$ for $0 \leq i \leq l-2$ using Proposition 4.3.

Step 6. Construct $\mathbf{R E C}_{\mathbf{l - 2}}$ using Proposition 4.4.

Step 7. $\quad$ Set $l=l-1$, and go to Step 4 .

There is some similarity between our algorithm and results of Schneider [Sch]; we do believe however the underlying algorithm to obtain the certificates is different from Schneider's program Sigma, although he did employ some version of Abramov's algorithm.

The subtle part of the above algorithm are steps 5 and 6 which compute the proper denominators for the certificates that appear in Equations (8). This is done using Propositions 4.3 and 4.4, which follow from Theorem 5.1, which are implemented in our improved denominator algorithm of Section 2.2.

Example 2.2. When $r=3$ the algorithm computes $\mathbf{R E C}_{\mathbf{i}}$ for $0 \leq i \leq 3$ and $\mathbf{R E C}_{\mathbf{i}, \mathbf{j}}$ for $0 \leq j<i \leq 3$ in the following order:

\section{$\mathrm{REC}_{3,0}, \mathrm{REC}_{3,1}, \mathrm{REC}_{3,2}, \mathrm{REC}_{3} \rightarrow \mathrm{REC}_{2} \rightarrow \mathrm{REC}_{2,0}, \mathrm{REC}_{2,1} \rightarrow \mathrm{REC}_{1} \rightarrow \mathrm{REC}_{1,0} \rightarrow \mathrm{REC}_{0}$}

A Maple implementation of the above algorithm is available at [GS2]. A Mathematica implementation will be developed later. A $q$-version of the above algorithm is possible and will also be developed later.

2.2. An improved universal denominator algorithm. In this section we present our universal denominator algorithm. Let $K$ denote a fixed field, which in applications it is the field of rational functions with rational coefficients in a finite set of variables.

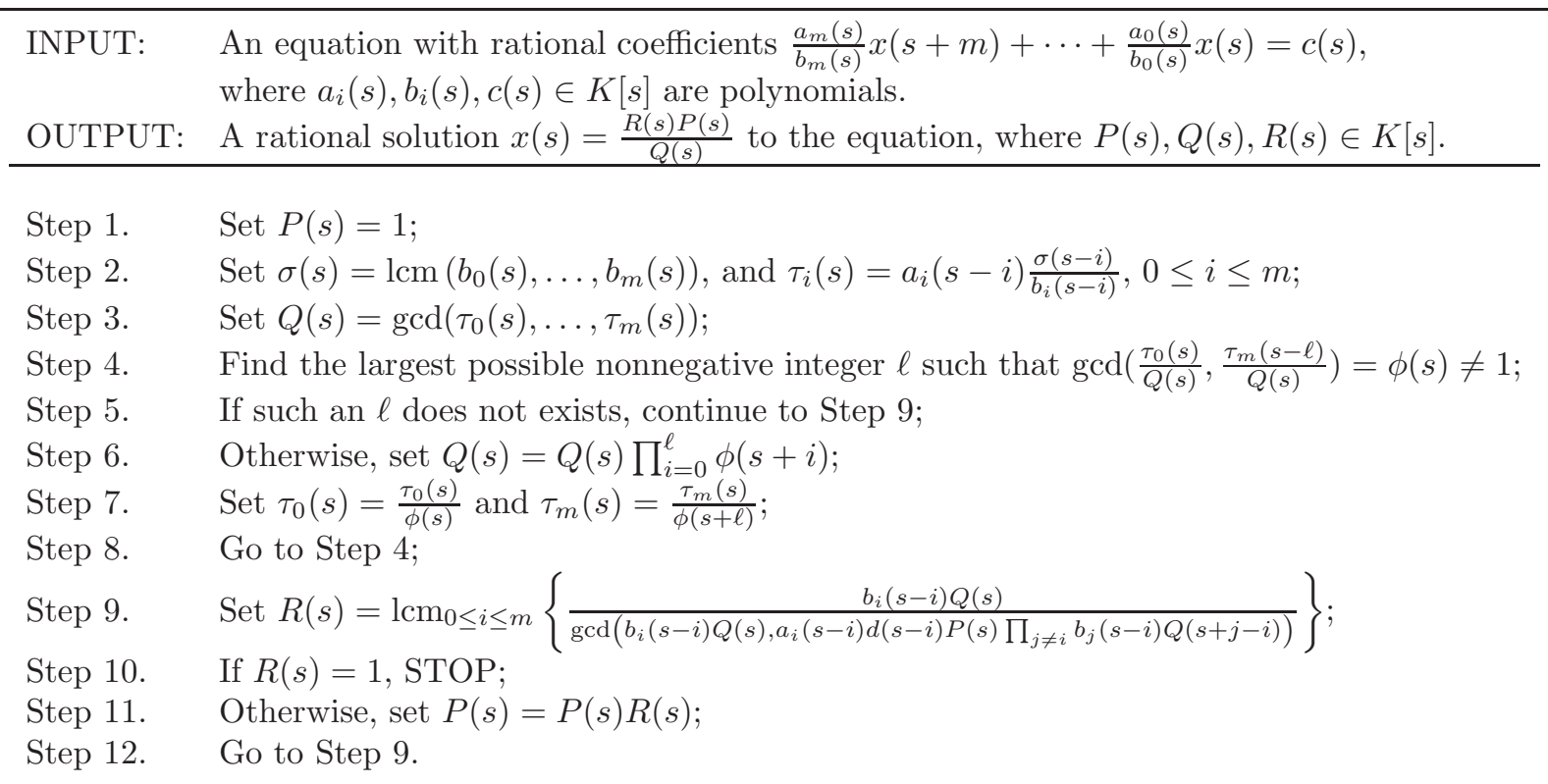

2.3. Plan of the proof. The structure of the paper is as follows. In Section 3, we explain the usage of the Maple program. We present a few examples and compare the results and performance against the programs discussed above. In Section 2, we introduce the terminology used in the paper, and present the general 
structure of the method as a sequence of steps. In Section 4, we prove the validity of each step of the structure, and also explain the method in detail. In Section 5, we prove a new algorithm that generates universal denominators with possibly less factors than those generated by Abramov's algorithm, that also partially predict the numerators for rational solutions to linear difference equations.

\section{UsE OF THE PROGRAM AND EXAMPLES}

Example 3.1. Define

$$
\begin{aligned}
f\left(n, k_{1}, k_{2}\right) & =(-1)^{n+k_{1}+k_{2}}\left(\begin{array}{l}
n \\
k_{1}
\end{array}\right)\left(\begin{array}{l}
n \\
k_{2}
\end{array}\right)\left(\begin{array}{c}
n+k_{1} \\
k_{1}
\end{array}\right)\left(\begin{array}{c}
n+k_{2} \\
k_{2}
\end{array}\right)\left(\begin{array}{c}
2 n-k_{1}-k_{2} \\
n
\end{array}\right) \\
g(n, k) & =\left(\begin{array}{l}
n \\
k
\end{array}\right) .
\end{aligned}
$$

We will prove that ([PWZ, Page 33] and [Sch])

$$
\sum_{k_{1}, k_{2}} f\left(n, k_{1}, k_{2}\right)=\sum_{k} g(n, k) .
$$

After running the program, both sides of the above equation are annihilated by the operator

$$
(n+2)^{3} N^{2}-2(2 n+3)\left(3 n^{2}+9 n+7\right) N-4(4 n+5)(4 n+3)(n+1) .
$$

Since they have the same initial conditions for $n=0,1$, the two sides agree for all natural numbers $n$.

Please see [GS2] for the syntax of input and output.

Example 3.2. Define

$$
f\left(n, k_{1}, k_{2}\right)=\left(\begin{array}{c}
n \\
k_{1}
\end{array}\right)\left(\begin{array}{c}
n \\
k_{2}
\end{array}\right)\left(\begin{array}{c}
n+k_{1} \\
k_{1}
\end{array}\right)\left(\begin{array}{c}
n+k_{2} \\
k_{2}
\end{array}\right)\left(\begin{array}{c}
2 n-k_{1}-k_{2} \\
n
\end{array}\right) .
$$

Please see [GS2] for complete information. The recursion for the multisum $\sum_{k_{1}, k_{2}} f\left(n, k_{1}, k_{2}\right)$ is of degree 4 .

Example 3.3. Define

$$
f\left(n, k_{1}, k_{2}, k_{3}\right)=(-1)^{n+k_{1}+k_{2}+k_{3}}\left(\begin{array}{l}
n \\
k_{1}
\end{array}\right)\left(\begin{array}{l}
n \\
k_{2}
\end{array}\right)\left(\begin{array}{c}
n \\
k_{3}
\end{array}\right)\left(\begin{array}{c}
n+k_{1} \\
k_{1}
\end{array}\right)\left(\begin{array}{c}
n+k_{2} \\
k_{2}
\end{array}\right)\left(\begin{array}{c}
n+k_{3} \\
k_{3}
\end{array}\right)\left(\begin{array}{c}
2 n-k_{1}-k_{2}-k_{3} \\
n
\end{array}\right) .
$$

Please see [GS2] for complete information. The recursion for the multisum $\sum_{k_{1}, k_{2}, k_{3}} f\left(n, k_{1}, k_{2}, k_{3}\right)$ is of degree 4 .

\section{Proof of the multisum Algorithm}

4.1. Two Lemmas. We fix a term $f(n, k)$ where $k=\left(k_{1}, \ldots, k_{r}\right)$, and consider a fixed variable $k_{v}$ and the corresponding operator $K_{v}$. The moduli are always $\left(k_{v+1}, \ldots, k_{r}\right)$, which we suppress for simplicity.

Lemma 4.1. If $\sum_{i=0}^{I} b_{i}\left(n, k_{1}, \ldots, k_{v}\right) K_{v}^{i} \equiv_{f} 0$ and $N+\sum_{i=0}^{I-1} a_{i}\left(n, k_{1}, \ldots, k_{v}\right) K_{v}^{i} \equiv_{f} 0$, then for any integer $m$ and rational functions $\left\{\alpha_{i}\left(n, k_{1}, \ldots, k_{v}\right)\right\}_{0 \leq i \leq m}$, there exist rational functions $\left\{\beta_{j}\left(n, k_{1}, \ldots, k_{v}\right)\right\}_{0 \leq j \leq I-1}$ so that

$$
\sum_{i=0}^{m} \alpha_{i}\left(n, k_{1}, \ldots, k_{v}\right) N^{i} \equiv \sum_{j=0}^{I-1} \beta_{j}\left(n, k_{1}, \ldots, k_{v}\right) K_{v}^{j}
$$

Furthermore, Equation (9) is a linear system of equations with unknowns $\left\{\beta_{j}\left(n, k_{1}, \ldots, k_{v}\right)\right\}$ and coefficients in the field $\mathbb{Q}\left(n, k_{1}, \ldots, k_{v}\right)$.

Proof. Since the operators are linear over the field $\mathbb{Q}\left(n, k_{1}, \ldots, k_{v}\right)$, we only need to show the result for $N^{m}$ for $m \geq 1$ by induction. 
The conclusion is true for $m=1$. Suppose it is true for $m-1$, i.e., $N^{m-1} \equiv_{f} \sum_{j=0}^{I-1} \gamma_{j}\left(n, k_{1}, \ldots, k_{v}\right) K_{v}^{j}$ for some $\left\{\gamma_{i}\right\}$. Then we have

$$
\begin{aligned}
N^{m} & \equiv_{f} \quad N\left(N^{m-1}\right) \equiv_{f} N\left(\sum_{j=0}^{I-1} \gamma_{j}\left(n, k_{1}, \ldots, k_{v}\right) K_{v}^{j}\right) \equiv_{f} \sum_{j=0}^{I-1} \gamma_{j}\left(n+1, k_{1}, \ldots, k_{v}\right) K_{v}^{j} N \\
& \equiv{ }_{f} \quad \sum_{j=0}^{I-1} \gamma_{j}\left(n+1, k_{1}, \ldots, k_{v}\right) K_{v}^{j}\left(-\sum_{i=0}^{I-1} a_{i}\left(n, k_{1}, \ldots, k_{v}\right) K_{v}^{i}\right) \equiv_{f} \sum_{i=0}^{I-1} \beta_{i}\left(n, k_{1}, \ldots, k_{v}\right) K_{v}^{i},
\end{aligned}
$$

for some rational $\beta_{i}\left(n, k_{1}, \ldots, k_{v}\right)$. The last equation is because the order of recursion in $k_{v}$ satisfied by $f$ is at most $I$.

Since the reduction of $N^{m}$ does not depend on $\left\{\alpha_{i}\left(n, k_{1}, \ldots, k_{v}\right)\right\}$, it follows that $\left\{\beta_{j}\left(n, k_{1}, \ldots, k_{v}\right)\right\}$ are linear functions of $\left\{\alpha_{i}\left(n, k_{1}, \ldots, k_{v}\right)\right\}$.

Lemma 4.2. Given $K_{v}^{p}+\sum_{i=0}^{p-1} a_{i}\left(n, k_{1}, \ldots, k_{v}\right) K_{v}^{i} \equiv_{f} 0$ and

$$
-\sum_{j=0}^{p-1} a_{p-1-j}\left(n, k_{1}, \ldots, k_{v}+j\right) b_{p-1}\left(n, k_{1}, \ldots, k_{v}+1+j\right)-b_{p-1}\left(n, k_{1}, \ldots, k_{v}\right)=\sum_{j=0}^{p-1} c_{p-1-j}\left(n, k_{1}, \ldots, k_{v}+j\right)
$$

where $\left\{c_{i}\left(n, k_{1}, \ldots, k_{v}\right)\right\}_{0 \leq i \leq p-1}$ and $b_{p-1}\left(n, k_{1}, \ldots, k_{v}\right)$ are rational functions. Define, for $0 \leq i<p-1$,

$$
\begin{aligned}
b_{i}\left(n, k_{1}, \ldots, k_{v}\right)= & b_{p-1}\left(n, k_{1}, \ldots, k_{v}-p+1+i\right)+\sum_{j=1}^{p-i-1} a_{i+j}\left(n, k_{1}, \ldots, k_{v}-j\right) b_{p-1}\left(n, k_{1}, \ldots, k_{v}-j+1\right) \\
& +\sum_{j=1}^{p-i-1} c_{i+j}\left(n, k_{1}, \ldots, k_{v}-j\right) .
\end{aligned}
$$

Then

$$
\sum_{i=0}^{p-1} c_{i}\left(n, k_{1}, \ldots, k_{v}\right) k_{v}^{i} \equiv_{f}\left(k_{v}-1\right) \sum_{i=0}^{p-1} b_{i}\left(n, k_{1}, \ldots, k_{v}\right) k_{v}^{i}
$$

Proof. From the definition of $b_{i}\left(n, k_{1}, \ldots, k_{v}\right)$, it is easy to check that

$$
\begin{aligned}
b_{i-1}\left(n, k_{1}, \ldots, k_{v}+1\right) & =b_{i}\left(n, k_{1}, \ldots, k_{v}\right)+a_{i}\left(n, k_{1}, \ldots, k_{v}\right) b_{p-1}\left(n, k_{1}, \ldots, k_{v}+1\right)+c_{i}\left(n, k_{1}, \ldots, k_{v}\right), \\
b_{0}\left(n, k_{1}, \ldots, k_{v}\right) & =-b_{p-1}\left(n, k_{1}, \ldots, k_{v}+1\right) a_{0}\left(n, k_{1}, \ldots, k_{v}\right)-c_{0}\left(n, k_{1}, \ldots, k_{v}\right) .
\end{aligned}
$$

It follows that 


$$
\begin{aligned}
& \left(K_{v}-1\right) \sum_{i=0}^{p-1} b_{i}\left(n, k_{1}, \ldots, k_{v}\right) K_{v}^{i} \quad \equiv_{f} \quad b_{p-1}\left(n, k_{1}, \ldots, k_{v}+1\right) K_{v}^{p}+\sum_{i=0}^{p-2} b_{i}\left(n, k_{1}, \ldots, k_{v}+1\right) K_{v}^{i+1} \\
& -\sum_{i=0}^{p-1} b_{i}\left(n, k_{1}, \ldots, k_{v}\right) K_{v}^{i} \\
& \equiv_{f} \quad-\sum_{i=0}^{p-1} b_{p-1}\left(n, k_{1}, \ldots, k_{v}+1\right) a_{i}\left(n, k_{1}, \ldots, k_{v}\right) K_{v}^{i} \\
& +\sum_{i=1}^{p-1}\left(b_{i-1}\left(n, k_{1}, \ldots, k_{v}+1\right)-b_{i}\left(n, k_{1}, \ldots, k_{v}\right)\right) K_{v}^{i}-b_{0}\left(n, k_{1}, \ldots, k_{v}\right) \\
& \equiv_{f} \quad-\sum_{i=0}^{p-1} b_{p-1}\left(n, k_{1}, \ldots, k_{v}+1\right) a_{i}\left(n, k_{1}, \ldots, k_{v}\right) K_{v}^{i} \\
& +\sum_{i=1}^{p-1}\left(a_{i}\left(n, k_{1}, \ldots, k_{v}\right) b_{p-1}\left(n, k_{1}, \ldots, k_{v}+1\right)+c_{i}\left(n, k_{1}, \ldots, k_{v}\right)\right) K_{v}^{i} \\
& +b_{p-1}\left(n, k_{1}, \ldots, k_{v}+1\right) a_{0}\left(n, k_{1}, \ldots, k_{v}\right)+c_{0}\left(n, k_{1}, \ldots, k_{v}\right) \\
& \equiv_{f} \quad \sum_{i=0}^{p-1} c_{i}\left(n, k_{1}, \ldots, k_{v}\right) K_{v}^{i} \text {. }
\end{aligned}
$$

Lemma 4.2 also appeared in [Sch] in a different form. It is included here for completeness of the proofs.

4.2. Two propositions for the algorithm. In this section we state and prove Propositions 4.3 and 4.4 which are used in our algorithm. Fix a term $f(n, k)$ where $k=\left(k_{1}, \ldots, k_{r}\right)$. Recall we set $k_{0}=n$ and $K_{0}=N$.

Proposition 4.3. Let $1 \leq v<r$. Given $f$-compatible operators $\mathbf{R E C}_{\mathbf{v}+\mathbf{1}}, \mathbf{R E C}_{\mathbf{v}}, \mathbf{R E C}_{\mathbf{v}+\mathbf{1}, \mathbf{u}}, \mathbf{R E C} \mathbf{C}_{\mathbf{v}+\mathbf{1}, \mathbf{v}}$ for $0 \leq u \leq v \leq r$, it is possible to construct an $f$-compatible operator $\mathbf{R E C}_{\mathbf{v}, \mathbf{u}}$ for $0 \leq u<v$ in Step 5 .

Proposition 4.4. Let $1 \leq v \leq r$. Given $f$-compatible operators $\mathbf{R E C}_{\mathbf{v}}, \mathbf{R E C}_{\mathbf{v}, \mathbf{v}-\mathbf{1}}$ for $0 \leq u \leq v$, it is possible to construct $f$-compatible operator $\mathbf{R E C}_{\mathbf{v}-\mathbf{1}}$ in Steps 3 and 6.

4.3. Proof of Proposition 4.3. Let

$\mathbf{R E C}_{\mathbf{v}+\mathbf{1}}:=K_{v+1}^{J}+\sum_{i=0}^{J-1} a_{i}\left(k_{0}, k_{1}, \ldots, k_{v+1}\right) K_{v+1}^{i} \quad$ and $\quad \mathbf{R E C}_{\mathbf{v}+\mathbf{1}} \equiv_{f} 0 \bmod \left(K_{v+2}-1, \ldots, K_{r}-1\right)$.

We can always divide the operator by the leading coefficient if it is not 1 , since it does not involve variables $k_{v+2}, \ldots, k_{r}$. Let us look for

$\left(1 \mathbb{R} \mathbf{E C}_{\mathbf{v}, \mathbf{u}}:=K_{u}+\sum_{i=0}^{I-1} \phi_{i}\left(k_{0}, k_{1}, \ldots, k_{v}\right) K_{v}^{i} \quad\right.$ and $\quad \mathbf{R E C}_{\mathbf{v}, \mathbf{u}} \equiv_{f} 0 \bmod \left(K_{v+1}-1, \ldots, K_{r}-1\right)$,

for some rational functions $\phi_{i}\left(k_{0}, k_{1}, \ldots, k_{v}\right)$. To prove the existence of $\mathbf{R E C}_{\mathbf{v}, \mathbf{u}}$, borrow the idea in the proof of [WZ] by solving

$$
\left[K_{u}+\sum_{i_{v}=0}^{U_{v}} \cdots \sum_{i_{m}=0}^{U_{m}}\left(\sigma_{i_{v} \cdots i_{m}}\left(k_{0}, k_{1}, \ldots, k_{v}\right) \prod_{l=v}^{m} K_{l}^{i_{l}}\right)\right] f\left(k_{0}, k_{1}, \ldots, k_{r}\right)=0
$$


with $\sigma_{i_{v} \ldots i_{m}}\left(k_{0}, k_{1}, \ldots, k_{v}\right)$ being the unknown rational functions. Divide both sides by the hypergeometric function $f\left(k_{0}, \ldots, k_{r}\right)$ to obtain an equation of rational functions. By comparing the coefficients of the powers of $k_{v+1}, \ldots, k_{r}$, we can set up a system of linear equations over the field $\mathbb{Q}\left(k_{0}, k_{1}, \ldots, k_{v}\right)$, whose unknowns are $\sigma_{i_{v} \cdots i_{m}}\left(k_{0}, k_{1}, \ldots, k_{v}\right)$. The number of unknowns is $\prod_{l=v}^{m}\left(U_{l}+1\right)$, while the number of equations, which equals the degree of the numerator in Equation (12), is proportional to $\left(\prod_{l=v}^{m} U_{l}\right)\left(\sum_{l=v}^{m} \frac{1}{U_{l}}\right)$. It follows that when $U_{v}, \ldots, U_{m}$ are large enough, we have more unknowns than equations in the system, which guarantees a nontrivial solution. Replacing $K_{v+1}, \ldots, K_{r}$ in Equation (12) with 1, we get a solution to Equation (11). The maximum power of $I-1$ on $K_{v}$ is ensured by the existence of a recursion of order $I$. The readers may also compare with [PWZ, Theorem 4.4.1] or [AZ, Theorem MZ] for a detailed discussion on the method in similar cases. With the proof of existence completed, we can introduce a new method to find the functions $\left\{\phi_{i}\right\}$ and $\left\{b_{j}\right\}$.

Reduce $K_{u}+\sum_{i=0}^{I-1} \phi_{i}\left(k_{0}, k_{1}, \ldots, k_{v}\right) K_{v}^{i}$ into $\sum_{i=0}^{J-1} c_{i}\left(k_{0}, k_{1}, \ldots, k_{v+1}\right) K_{v+1}^{i}$ for some rational $c_{i}\left(k_{0}, k_{1}, \ldots, k_{v+1}\right)$, using Lemma 4.1 below. This implies that

$$
\sum_{i=0}^{J-1} c_{i}\left(k_{0}, k_{1}, \ldots, k_{v+1}\right) K_{v+1}^{i} \equiv_{f}\left(K_{v+1}-1\right)\left(\sum_{i=0}^{J-1} b_{i}\left(k_{0}, k_{1}, \ldots, k_{v+1}\right) K_{v+1}^{i}\right)
$$

Since the coefficient of $K_{v+1}^{J}$ is 1 in (10), it follows from Lemma 4.2 below that we only need to find $b_{J-1}\left(k_{0}, k_{1}, \ldots, k_{v+1}\right)$ such that

$$
\begin{array}{r}
-\sum_{j=-1}^{J-1} a_{J-1-j}\left(k_{0}, k_{1}, \ldots, k_{v+1}+j\right) b_{J-1}\left(k_{0}, k_{1}, \ldots, k_{v+1}+1+j\right) \\
=\sum_{j=0}^{J-1} c_{J-1-j}\left(k_{0}, k_{1}, \ldots, k_{v+1}+j\right) .
\end{array}
$$

In the equation, $\left\{a_{i}\left(k_{0}, k_{1}, \ldots, k_{v+1}\right)\right\}_{0 \leq i \leq J-1}$ are known; $b_{J-1}$ is a rational function over $k_{0}, \ldots, k_{v+1}$ over the field $\mathbb{Q}\left(k_{0}, k_{1}, \ldots, k_{v}\right)$; and $\left\{c_{i}\right\}_{0 \leq i \leq J-1}$ are linear over $\left\{\phi_{j}\right\}_{0 \leq j \leq I-1}$. So the right-hand side can be written as $\frac{\sum_{j=0}^{I-1} U_{j}\left(k_{0}, k_{1}, \ldots, k_{v+1}\right) \phi_{j}\left(k_{0}, k_{1}, \ldots, k_{v}\right)}{V\left(k_{0}, k_{1}, \ldots, k_{v+1}\right)}$, with polynomials $\phi_{j}\left(k_{0}, k_{1}, \ldots, k_{v}\right)$ unknown; and $U_{j}\left(k_{0}, k_{1}, \ldots, k_{v+1}\right)$ and $V\left(k_{0}, k_{1}, \ldots, k_{v+1}\right)$ known. Multiply both sides of Equation $(13)$ by $V\left(k_{0}, k_{1}, \ldots, k_{v+1}\right)$ to obtain

$$
\begin{array}{r}
\sum_{j=-1}^{J-1}-a_{J-1-j}\left(k_{0}, k_{1}, \ldots, k_{v+1}+j\right) b_{J-1} \\
\left(k_{0}, k_{1}, \ldots, k_{v+1}+1+j\right) V\left(k_{0}, k_{1}, \ldots, k_{v+1}\right) \\
=\sum_{j=0}^{I-1} U_{j}\left(k_{0}, k_{1}, \ldots, k_{v+1}\right) \phi_{j}\left(k_{0}, k_{1}, \ldots, k_{v}\right) .
\end{array}
$$

In the above equation, consider $b_{J-1}\left(k_{0}, \ldots, k_{v+1}\right) \in \mathbb{Q}\left(k_{0}, \ldots, k_{v}\right)\left(k_{v+1}\right)$, and apply Theorem 5.1 to the field $K=\mathbb{Q}\left(k_{0}, \ldots, k_{v}\right)$ and the variable $s=k_{v+1}$. It follows that we can write

$$
b_{J-1}\left(k_{0}, k_{1}, \ldots, k_{v+1}\right)=\frac{R\left(k_{0}, k_{1}, \ldots, k_{v+1}\right) P\left(k_{0}, k_{1}, \ldots, k_{v+1}\right)}{Q\left(k_{0}, k_{1}, \ldots, k_{v+1}\right)},
$$

with polynomials $R\left(k_{0}, k_{1}, \ldots, k_{v+1}\right) \in \mathbb{Q}\left[k_{0}, \ldots, k_{v+1}\right]$ and $Q\left(k_{0}, k_{1}, \ldots, k_{v+1}\right) \in \mathbb{Q}\left[k_{0}, \ldots, k_{v+1}\right]$ known, and $P\left(k_{0}, k_{1}, \ldots, k_{v+1}\right) \in \mathbb{Q}\left[k_{0}, \ldots, k_{v+1}\right]$ unknown. By multiplying both sides by the common denominator of the left-hand side, and comparing the degree of $k_{v+1}$, we can determine the degree of $k_{v+1}$ in $P\left(k_{0}, k_{1}, \ldots, k_{v+1}\right)$, say, $L$. By writing $P\left(k_{0}, k_{1}, \ldots, k_{v+1}\right)$ as $\sum_{i=0}^{L} \psi_{i}\left(k_{0}, k_{1}, \ldots, k_{v}\right) k_{v+1}^{i}$, plugging it back into Equation (14), and comparing the coefficients of powers of $k_{v+1}$, we can set up a system of linear equations with $\left\{\phi_{j}\right\}_{0 \leq j \leq I-1}$ and $\left\{\psi_{i}\right\}_{0 \leq i \leq L}$ as unknowns. The system is guaranteed to have a nontrivial solution because of the existence of the recursion. 
4.4. Proof of Proposition 4.4. The existence of the recursion can be proved in a way similar to Theorem 4.3. And the method of the new algorithm is also the same. Basically we again rewrite the left-hand side of the equations into powers of $K_{v}$, compare their coefficients on both sides, and solve the resulting linear equations. Details are omitted.

\section{Proof of the Universal Denominator Algorithm}

In this section we state and prove Theorem 5.1 which determines the denominator and partially the numerator of the rational function $b_{J-1}$ in Equation (14). This is crucial for the performance of the algorithm as a whole, because it reduces the number of variables and number of equations in the final system of linear equations to be solved. The most straight-forward guess for the denominator $b_{J-1}$ in Equation (14), i.e., the denominator of the right-hand side of the equation, will give us an algorithm whose performance is compatible to that of Sister Celine's method on a single step. Theorem 5.1 also improves Abramov's universal denominator $[\mathrm{Ab}]$.

Let $K$ denote a field, which for our applications it will be the field of rational functions with rational coefficients in a finite set of variables. Let $s$ denote a fixed variable that does not appear in $K$. As usual, if $p(s), q(s) \in K[s]$ are polynomials, then we write $p(s) \mid q(s)$ if $p(s)$ divides $q(s)$.

Consider the equation

$$
\sum_{i=0}^{m} \frac{a_{i}(s)}{b_{i}(s)} x(s+i)=c(s),
$$

where $a_{i}(s), b_{i}(s), c(s) \in K[s]$ are polynomials, and $\operatorname{gcd}\left(a_{i}, b_{i}\right)=1$. Define

$$
\begin{aligned}
\sigma(s) & =\operatorname{lcm}\left(b_{i}(s) \mid 0 \leq i \leq m\right), \\
\tau_{i}(s) & =\frac{a_{i}(s-i)}{b_{i}(s-i)} \sigma(s-i), 0 \leq i \leq m, \\
\hat{\tau}(s) & =\operatorname{gcd}\left(\tau_{0}(s), \ldots, \tau_{m}(s)\right)
\end{aligned}
$$

and

$$
\begin{aligned}
Q(s)= & \hat{\tau}(s) \prod_{i=0}^{I} \prod_{j=0}^{J_{i}} \phi_{i}(s+j), \text { where } \phi_{i}(s)\left|\frac{\tau_{0}(s)}{\hat{\tau}(s)}, \phi_{i}\left(s+J_{i}\right)\right| \frac{\tau_{r}(s)}{\hat{\tau}(s)}, \\
& \text { where each } J_{i} \text { is the maximum of such numbers for the function } \phi_{i}, \\
& \text { and the outer product is over all such } \phi_{i}, \\
R(s)= & \operatorname{lcm}_{0 \leq i \leq m}\left\{\frac{b_{i}(s-i) Q(s)}{\operatorname{gcd}\left(b_{i}(s-i) Q(s), a_{i}(s-i) \prod_{j \neq i} b_{j}(s-i) Q(s+j-i)\right)}\right\}
\end{aligned}
$$

Obviously, $R(s), Q(s) \in K[s]$ are polynomials.

Theorem 5.1. With the above conventions, every rational solution of (15) has the form

where $P(s) \in K[s]$ is a polynomial.

Proof. Suppose $x(s)=\frac{A(s)}{B(s)}$, with $\operatorname{gcd}(A(s), B(s))=1$. Then

$$
\sum_{i=0}^{m} \frac{a_{i}(s) \sigma(s)}{b_{i}(s)} \frac{A(s+i)}{B(s+i)}=c(s) \sigma(s) .
$$

So

$$
\sum_{i=0}^{m} \frac{a_{i}(s)}{B(s+i)} \frac{A(s+i) \sigma(s)}{b_{i}(s)}=c(s) \sigma(s)
$$




$$
c(s) \sigma(s) \prod_{j=0}^{m} B(s+j)=\sum_{i=0}^{m} A(s+i) \tau_{i}(s+i) \prod_{j \neq i} B(s+j) .
$$

Since $\tau_{i}(s)$ are polynomials for all $i$, it follows that

$$
B(s+i) \mid A(s+i) \tau_{i}(s+i) \prod_{j \neq i} B(s+j) .
$$

Since $\operatorname{gcd}(A(s), B(s))=1$, it follows that

$$
B(s) \mid \tau_{i}(s) \prod_{j \neq i}^{m} B(s+j-i) .
$$

Write $B(s)=\prod_{i=0}^{U} \prod_{j=0}^{V_{i}} f_{i}(s+j) \prod_{j=0}^{W} g_{j}(s)$, where $U, V_{i}, W$ are constants; and $\operatorname{gcd}\left(g_{i}(s), g_{j}(s+L)\right)=1$ for any $i, j, L$; and $\operatorname{gcd}\left(g_{j}(s), f_{i}(s+l)\right)=1$ for any $i, j$, and $-m \leq l \leq m$. We call the functions $g_{j}(s)$ singletons, and $\left\{f_{i}(s+j)\right\}_{0 \leq j \leq V_{j}}$ chains, in which $f_{i}(s)$ are the heads of chains, and $f_{i}\left(s+V_{i}\right)$ the tails of chains. So we are writing $B(s)$ uniquely as a product of chains and singletons.

There are two cases:

Case I: The tail of one chains is always far apart from the head of another in $B(s)$, i.e., $\operatorname{gcd}\left(f_{i}(s+\right.$ $\left.\left.V_{i}\right), f_{j}(s+v)\right)=1$ for all $0 \leq i, j \leq U$ and $-m \leq v \leq m$. Then

$$
\begin{array}{r|c}
\prod_{i=0}^{U} f_{i}(s) \prod_{j=0}^{W} g_{j}(s)=\frac{B(s)}{\operatorname{gcd}\left(B(s), \prod_{j=1}^{m} B(s+j)\right)} & \operatorname{gcd}\left(B(s), \tau_{0}(s)\right), \\
\prod_{i=0}^{U} f_{i}\left(s+V_{i}\right) \prod_{j=0}^{W} g_{j}(s)=\frac{B(s)}{\operatorname{gcd}\left(B(s), \prod_{j=1}^{m} B(s-j)\right)} & \operatorname{gcd}\left(B(s), \tau_{m}(s)\right), \\
\prod_{j=0}^{W} g_{j}(s)=\frac{B(s)}{\operatorname{gcd}\left(B(s), \prod_{j \neq i} B(s+j-i)\right)} & \operatorname{gcd}\left(B(s), \tau_{i}(s)\right), i \neq 0, m .
\end{array}
$$

Thus the singletons have the property

$$
\prod_{j=0}^{W} g_{j}(s) \mid \operatorname{gcd}\left(\tau_{i}, 0 \leq i \leq m\right) .
$$

At the same time, the heads of the chains $f_{i}(s)$ in $B(s)$ are factors of $\tau_{0}$, and the tails $f_{i}\left(s+V_{i}\right)$ factors of $\tau_{r}$. Therefore each chain in $B(s)$ factors $\prod_{j=0}^{J_{\ell}} \phi_{\ell}(s+j)$ for some $\ell$. Recalling the definition of $Q(s)$ from Equation (16), it follows that $B(s)$ divides $Q(s)$.

Case II: The heads and tails of chains are close, i.e., $\operatorname{gcd}\left(f_{i}\left(s+V_{i}\right), f_{j}(s+v)\right) \neq 1$ for some $0 \leq i, j \leq U$ and $-m \leq v \leq m$. In this case, $\prod_{i=0}^{I} \prod_{j=0}^{J_{i}} \phi_{i}(s+j)$ will contain a chain whose head is $f_{i}(s)$ and tail is $f_{j}\left(s+V_{j}\right)$ in $Q(s)$. This is a longer chain than what $B(s)$ really needs, but it still guarantees that $B(s)$ divides $Q(s)$.

So far, this proves that $x(s)=\frac{A(s)}{Q(s)}$ where $A(s) \in K[s]$ is a polynomial. To finish the proof, it suffices to show that $R(s)$ (given by Equation (17)) divides $A(s)$. Since

$$
\sum_{i=0}^{m} \frac{a_{i}(s)}{b_{i}(s)} \frac{A(s+i)}{Q(s+i)}=c(s),
$$


with $a_{i}, b_{i}, c$ polynomials, any polynomial factor that appears only once in the $m+1$ denominators on the left-hand side must also divide the corresponding numerator, which means

$$
\frac{b_{i}(s) Q(s+i)}{\operatorname{gcd}\left(b_{i}(s) Q(s+i), a_{i}(s) \prod_{j \neq i} b_{j}(s) \prod_{j \neq i} Q(s+j)\right)} \mid A(s+i) .
$$

Remark 5.1. When $m=1$, Theorem 5.1 becomes Gosper's algorithm. Recall that Gosper's algorithm tries to find rational solution $x(s)$ such that

$$
\frac{a(s) c(s+\ell)}{b(s) c(s)} x(s+1)-x(s)=1
$$

for some integer $\ell$. Based on our propositions, we get a chain $\prod_{i=0}^{\ell-1} c(s+i)$ as the denominator and $b(s-1)$ as part of the numerator, which agrees with Gosper's result.

Remark 5.2. Abramov's universal denominator treats the singletons in Theorem 5.1 as chains of length 1 , and then tries to find all chains. However, by picking singletons out first, we reduce the possibility of generating redundant chains in the denominator, because factors in the leading coefficient may mingle with the singletons and generate unwanted factors in chains. We illustrate the effect by example.

Example 5.3. This is Example 1 in $[\mathrm{Ab}]$.

$$
\begin{array}{r}
(n+4)(2 n+1)(n+2) x(n+3)-(2 n+3)(n+3)(n+1) x(n+2)+n(n+2)(2 n-3) x(n+1) \\
-(n-1)(2 n-1)(n+1) x(n)=0 .
\end{array}
$$

Abramov's algorithm gives the denominator $u(n)=n^{3}-n$ for all rational function solutions $x(n) \in \mathbb{Q}(n)$ of the above equation, and computes the general polynomial solution $C\left(2 n^{2}-3 n\right)$. However our algorithm finds two singletons $(n+1)(n-1)$ and no chains. So the denominator is $Q(n)=n^{2}-1$, which strictly divides $u(n)$.

Example 5.4. In one of the intermediate steps for Example 3.3, we get

$$
\begin{aligned}
x\left(n, k_{1}, k_{2}\right) & +\frac{-\left(2 k_{2}^{2}+k_{2}+4 k_{2} k_{1}-6 k_{2} n-3 n+k_{1}+3 n^{2}-6 k_{1} n+2 k_{1}^{2}\right)\left(n+k_{2}+2\right)\left(-n+k_{2}+1\right)}{\left(k_{2}+2\right)^{2}\left(k_{1}+1-n+k_{2}\right)\left(k_{1}-3 n+k_{2}\right)} x\left(n, k_{1}, k_{2}+1\right) \\
& +\frac{\left(k_{1}+1-n+k_{2}\right)^{2}\left(n+k_{2}+3\right)\left(n+k_{2}+2\right)\left(-n+k_{2}+2\right)\left(-n+k_{2}+1\right)}{\left(k_{2}+3\right)^{2}\left(k_{2}+2\right)^{2}\left(k_{1}+2-n+k_{2}\right)\left(k_{1}-3 n+k_{2}+1\right)} x\left(n, k_{1}, k_{2}+2\right) \\
& =\frac{c\left(n, k_{1}, k_{2}\right)}{\left(n+k_{2}+1\right)\left(-n+k_{2}\right) \prod_{j=0}^{2}\left[\left(k_{1}-3 n+k_{2}+j\right)\left(k_{1}-n+k_{2}+1+j\right)\left(k_{1}+1+j\right)^{2}\right]},
\end{aligned}
$$

to solve for $x\left(n, k_{1}, k_{2}\right)$ with $c\left(n, k_{1}, k_{2}\right)$ a polynomial. After multiplying both sides by the denominator of the right-hand side, we find four singletons $\left(n+k_{2}+1\right)\left(-n+k_{2}\right)\left(k_{1}-3 n+k_{2}\right)\left(k_{1}-n+k_{2}+1\right)$; no chain in the denominator of $x\left(n, k_{1}, k_{2}\right)$; and $k_{2}^{2}\left(k_{2}+1\right)^{2}$ as factors of the numerator of $x\left(n, k_{1}, k_{2}\right)$. Hence

$$
x\left(n, k_{1}, k_{2}\right)=\frac{k_{2}^{2}\left(k_{2}+1\right)^{2}}{\left(n+k_{2}+1\right)\left(-n+k_{2}\right)\left(k_{1}-3 n+k_{2}\right)\left(k_{1}-n+k_{2}+1\right)} P\left(n, k_{1}, k_{2}\right),
$$

where $P\left(n, k_{1}, k_{2}\right)$ is a polynomial.

Our method keeps finding the best possible denominators in all the steps of the examples discussed in the paper.

\section{REFERENCES}

[Ab] S.A. Abramov, Rational solutions of linear difference and q-difference equations with polynomial coefficients, Proc. ISSAC'95, ACM Press, 1995, 285-289.

[AZ] M. Apagodu and D. Zeilberger, Multi-Variable Zeilberger and Almkvist-Zeilberger Algorithms and the Sharpening of Wilf-Zeilberger Theory, Adv. Appl. Math 37 (2006) 139-152. 
[Fas] Sister Mary Celiner Fasenmyer, Some generalized hypergeometric polynomials, Ph.D. dissertation, University of Michigan, November, 1945.

[GS1] S. Garoufalidis and X. Sun, The non-commutative A-polynomial of twist knots, preprint 2008 arXiv:0802.4074.

[GS2] and Computer data available at ww. math.gatech.edu/ stavros/publications.html.

[Gos] R. W. Gosper, Decision procedures for indefinite hypergeometric summation, Proc. Nat. Acad. Sci, 75 (1978), 40-42.

[PSc] P. Paule and C. Schneider, Creative telescoping for hypergeometric double sums, Preprint, 2005.

[PSh] P. Paule and M. Schorn, A Mathematica version of Zeilberger's algorithm for proving binomial coefficient identities, J. Symbolic Comput. 20 (1995) 673-698.

[PWZ] M. Petkovšek, H.S. Wilf and D. Zeilberger, $A=B$, A.K. Peters, Ltd., Wellesley, MA 1996.

[Sch] C. Schneider, A new Sigma approach to multi-summation, Adv. Appl. Math 34 (2005) 740-767.

[Weg] K. Wegschaider, Computer generated proofs of binomial multi-sum identities, Diploma thesis, RISC Linz, Johannes Kepler University, May 1997.

[WZ] H. Wilf and D. Zeilberger, An algorithmic proof theory for hypergeometric (ordinary and q) multisum/integral identities, Inventiones Math. 108 (1992) 575-633.

[Ze] D. Zeilberger, Sister Celine's technique and its generalizations, J. Math. Anal. Appl. 85 (1982) 114-211.

School of Mathematics, Georgia Institute of Technology, Atlanta, GA 30332-0160, USA

http: //www. math. gatech.edu/ stavros

E-mail address: stavros@math.gatech.edu

Department of Mathematics, Tulane University, 6823 St. Charles Ave, New Orleans, la 70118, USA

http://www . math.tulane.edu/ $\sim$ xsun1

E-mail address: xsun1@tulane.edu 